\title{
IMAGES OF ASTROPHYSICAL MASERS AND THEIR VARIABILITY IN A TURBULENT MEDIUM: THE $25 \mathrm{GHz}$ METHANOL MASERS
}

\author{
A. M. SobOLEV ${ }^{1}$ \\ Astronomical Observatory, Ural State University, Lenin Street 51, Ekaterinburg 620083, Russia; Andrej.Sobolev@usu.ru \\ W. D. WATSON \\ Department of Physics, University of Illinois, 1110 West Green Street, Urbana, IL 61801; w-watson@uiuc.edu \\ AND \\ V. A. OKOROKOV \\ Chelyabinsk State University, Bratiev Kashirinykh Street 129, Chelyabinsk 454021, Russia; okr@csu.ru \\ Received 2002 September 5; accepted 2003 February 25
}

\begin{abstract}
We examine the influence of chance coherences in turbulent velocities for the creation of the images of astrophysical masers. Images are computed that represent the numerous masing features that are commonly observed in an extended masing region. Changes as a function of Doppler velocity can be seen in the appearance of these images. Variability in the images also is evident as a result of the evolution of the turbulent velocities with time. Representative turbulent velocity fields are obtained by standard methods involving the statistical sampling of Fourier components, and the medium is treated as incompressible for computing the time evolution of the turbulence. Comparisons with observational data focus on the $25 \mathrm{GHz}$ methanol masers, for which there is evidence that their structure may be caused mainly by coherences in the velocities.
\end{abstract}

Subject headings: ISM: molecules — masers — turbulence

\section{INTRODUCTION}

Irregular velocity fields occur in the diffuse gas for a wide range of environments in astronomy and for a wide range of physical conditions. Because of the "exponential sensitivity" of maser radiation to optical depth, it is likely that even small irregularities in the velocities of the gas play a key role in selecting the Doppler velocities at which strong maser emission is observed. These coherences in velocity are a plausible way to understand the tendency for maser emission to appear as bright spots. The possible influence of turbulence on our understanding of various features of astrophysical masers has been explored in a number of previous investigations, including Deguchi (1982), Walker (1984), Gwinn (1994a), Wallin, Watson, \& Wyld (1998, 1999), Wiebe \& Watson (1998), Sobolev, Wallin, \& Watson (1998, hereafter Paper I), and Imai, Deguchi, \& Sasao (2002).

The key feature of the $25 \mathrm{GHz}$ methanol masers that makes them useful for our analysis is the similarity of the physical parameters of the gas such as temperature, density, and the relative abundance of methanol that are derived from the maser line data (see, e.g., Sobolev \& Strel'nitskii 1983; Johnston et al. 1992 [hereafter JGS92]; Zeng, Lou, \& Li 1987) with the values of these quantities that are derived from thermal lines (Turner 1991; Sutton \& Herbst 1988; Kalenskii et al. 1997). Hence, the appearance of the $25 \mathrm{GHz}$ masers as spots is unlikely to be due to a form of clumping in which the values of these physical parameters are significantly different from their values in the general environment. It is then plausible that their appearance as spots is due mainly to chance coherences of turbulent velocities. Examining this possibility is the goal of our investigation.

\footnotetext{
${ }^{1}$ Visiting Scholar, Department of Physics, University of Illinois, 1110 West Green Street, Urbana, IL 61801.
}

The $25 \mathrm{GHz}$ masers are found in several sources in our Galaxy, indicating that they may be widespread (Menten et al. 1986). However, at present only one source is known (Ori-KL core in OMC-1) where these masers display a truly high flux density (about 200 Jy; see, e.g., Menten et al. 1988 and JGS92).

In Paper I it was shown that modeling the maser environment as a slab of gas with a turbulent velocity field reproduces the general properties of the Ori-KL $25 \mathrm{GHz}$ masers. The maser excitation was assumed to be constant within the slab, and the masers were assumed to be unsaturated. To match the observational data, it was found that the spectrum of the velocity distribution should be somewhat steeper than the Kolmogorov power law. In this model, the brightness of the maser region is strongly dependent on the orientation of the slab with respect to the observer. The highest brightnesses are achieved when the layer is seen edge-on. Since the probability for such an orientation is relatively low, it is understandable that we see masers with high brightness only in Ori-KL.

After our original investigation, observational information at high angular resolution on the images and on the time variability of the $25 \mathrm{GHz}$ maser spots in Ori-KL was published (Johnston et al. 1997, hereafter JGW97). In addition, a computer code for the calculation of time variations in the turbulent velocity field became available to us (see Wallin et al. 1999). These advances have provided the opportunity for further comparisons between observational data and the implications of a model in which the masing occurs in a turbulent slab.

\section{SUMMARY OF THE OBSERVATIONS}

Refined and extensive observational information about the high spatial resolution $(0.07)$ images of the $25 \mathrm{GHz}$ methanol masers associated with Ori-KL has been 
published by JGW97. The data provided by JGS92 were obtained with a more coarse spatial resolution $\left(3^{\prime \prime}\right)$. Because of the poor atmospheric conditions, JGW97 were able to detect only the brightest spots of the JGS92 sample.

The observations by JGW97 show that masers that appear to be single spots with the $3^{\prime \prime}$ beam are resolved into several spots in the observations with a 0.07 beam. The maser images obtained by JGW97 show substantial differences in their spatial distributions from one velocity to another, even for cases in which the velocities are separated by less than a line width. The spots in the JGW97 sample have sizes in the range $22-56$ AU and brightness temperatures from $2 \times 10^{6}$ to $3 \times 10^{7} \mathrm{~K}$. The sizes of the maser spots were found to increase with decreasing flux.

JGW97 observed the $25 \mathrm{GHz}$ masers of Ori-KL in two epochs separated by about 1 year (1990 March and 1991 July). For the most of the spots, the relative positions and fluxes were found to be about the same. However, the brightest maser spot was found to decrease its total flux density 1.6 times on the timescale of about a year. JGW97 noticed changes in the spatial distribution of the emission of that spot. The location of the peak shifted while the centroid remained at the same position. Two spots had disappeared at the second observational epoch. Comparison with the JGS92 data obtained in 1988 August indicates considerable variability as well.

On the other hand, observations of Menten et al. (1988) with the $100 \mathrm{~m}$ telescope show that the flux from the region as a whole does not change in magnitude nor in its spectral properties on the timescale of a year. These data rule out variations exceeding 20\% between 1986 February and 1987 March, and are consistent with the earlier data of Hills, Pankonin, \& Landecker (1974).

\section{BASIC PREMISES}

\subsection{Model of the Source}

The essential idealizations are the same as in our earlier investigation (Paper I) and are described in detail in Wallin et al. (1998). Representative turbulent velocity fields (or "realizations") are created by statistical sampling utilizing a Kolmogorov-like power spectrum for the Fourier components and a Gaussian distribution for the amplitudes. Such methods are standard. They create fields in a plausible manner that have the desired power spectrum in Fourier space and the desired autocorrelation (or structure) function for the velocities in coordinate space. In Paper I and here, we find that a spectrum that is somewhat steeper than that of Kolmogorov leads to results that tend to agree better with observations and with at least some numerical simulations (see Watson, Wiebe, \& Crutcher 2001). Thus, we use a power spectrum $k^{-7 / 3}$ instead of the $k^{-5 / 3}$ of the Kolmogorov law. The "structure function" (e.g., Frisch 1995), which follows from the power spectrum, then varies as (separation $)^{4 / 3}$ and is also steeper than the Kolmogorov variation, which is proportional to (separation) ${ }^{2 / 3}$. We do not attempt to incorporate any information about variations in the density or in the excitation. These are treated as constant.

As discussed in Paper I, the rectangular grid of points at which the velocities are created by this method is uniformly spaced. The size of the masing region in Ori-KL is about $10^{\prime \prime} \times 40^{\prime \prime}$ (JGS92). In order to make calculations with an angular resolution that is comparable to that of the JGW97 observations, we used $512 \times 128$ grid points for the face and 512 grid points for the depth of the volume. A cutoff is introduced when the wavelengths of the Fourier components are equal to the length of the smallest side of the rectangular volume, so that the power at longer wavelengths is severely reduced. The turbulence is then introduced at wavelengths near this maximum wavelength. At the other extreme, the shortest wavelength in the calculations is set as equal to twice the separation between the grid points. An overall constant in the power spectrum is adjusted so that the value for the rms turbulent velocities $\sigma_{t}$ is about five thermal breadths. With the assumption that the thermal breadth $v_{\text {th }}$ is equal to $0.22 \mathrm{~km} \mathrm{~s}^{-1}$, the computations reproduce the observed spread in the velocities of the maser features (Paper I).

The masers are treated as unsaturated so that it only is necessary to compute the optical depths. Numerical integrations are performed along the paths of rays that are perpendicular to the appropriate surface of the rectangular volume of grid points. If the $(y, z)$ plane is designated as the surface, the optical depth $\tau(v, y, z)$ for the ray emerging from the location $(y, z)$ with a frequency corresponding to Doppler velocity $v$ is

$$
\tau(v, y, z)=\left(\frac{\tau_{0}}{N_{x}}\right) \sum_{i} \exp \left[-\frac{\left(v-v_{t}^{i}\right)^{2}}{v_{\text {th }}^{2}}\right] .
$$

Here, $N_{x}$ is the number of grid points along the $x$-axis, $v_{t}^{i}$ is the turbulent velocity at grid point $i, \tau_{0}$ is an optical depth parameter (the optical depth at line center if there were no turbulence), and the sum is over the grid points along the ray.

Since $v_{\text {th }}$ refers to the thermal velocity of methanol molecules (as can be seen in eq. [1]) and the sound velocity is governed by the motion of the dominant species (here $\mathrm{H}_{2}$ ), the choice $\sigma_{t}=5 v_{\text {th }}$ corresponds to 1.7 times the sound velocity of the gas. Shocks would occur in an actual gas with this supersonic, turbulent velocity and would, strictly speaking, probably give rise to variations in the temperature, density, and composition of the gas. The results that we obtain from our idealized calculations in which these variations are ignored can, nevertheless, be indicative since the turbulence is only mildly supersonic and the pumping of the maser is not extremely sensitive to modest variations in temperature and density.

\subsection{Identification of Maser Spots}

In this investigation, we are considering images of numerous maser spots that have a wide range of fluxes. Identifying relatively faint maser spots in the three-dimensional $(v, y, z)$ space by hand is impractical. Thus, we used an automatic procedure to identify maser spots that is based on the definition of a maser spot as a maximum within a rectangular volume of $(v, y, z)$ space. We designate that volume as the spot vicinity. To identify maser spots in our model, we used a rectangular volume consisting of five grid points on a side in the spatial coordinates and $2 v_{\text {th }}$ in velocity as the spot vicinity. This choice allowed us to identify maser spots with a wide range of fluxes.

\subsection{Time Variability}

One of the main properties of the masers is the noticeable change of their characteristics with time. In this study, we 
examine whether the evolution of the turbulent velocity field can lead to a time variability that is similar to that of the observational data. In the same way as for our study of the images (Paper I), we examine the evolution of turbulence in the idealized case in which the changes in the masing medium are determined by the velocity field alone, i.e., without incorporating variations in the density, temperature, etc. This, again, corresponds to treating the masing medium as an incompressible fluid.

To treat the time evolution of the turbulent velocity field, we use a code that is based on the method of Chen \& Shan (1992). The code solves the Navier-Stokes equation

$$
\frac{d \boldsymbol{v}}{d t}=\boldsymbol{v}(\boldsymbol{\nabla} \cdot \boldsymbol{v})-\nu(\boldsymbol{\nabla})^{2} \boldsymbol{v}
$$

where $\boldsymbol{v}$ is the dimensionless velocity (velocity scaled by thermal line breadth, $v_{\mathrm{th}}$ ). The spatial coordinates are scaled by the length of the smallest side $L$ of the rectangular volume, the viscosity $\nu$ is scaled by the product $v_{\text {turb }} L$, and the time is scaled by $L / v_{\text {turb }}=\left(9 \times 10^{16} \mathrm{~cm}\right) /\left(5 \times 0.22 \mathrm{~km} \mathrm{~s}^{-1}\right)=$ $2.5 \times 10^{4} \mathrm{yr}$, where $L=9 \times 10^{16} \mathrm{~cm}$ corresponds to $10^{\prime \prime}$ at the distance of OMC-1. For the studies of the current paper it is sufficient to trace the evolution of the velocity field over intervals of dimensionless time up to $10^{-4}$ (see discussion of scaling factors in the subsequent sections).

As a measure of the ability of the code to describe the evolution of the turbulence, we note that the main statistical property - the dispersion of the velocity field - changes by less than $0.05 \%$ in a dimensionless time period of $10^{-4}$. During periods of this duration, we have also examined the behavior of other properties of the maser emission model which are most important for our study. These are the total flux from the maser region (i.e., the emission integrated over field of view and over all velocities) and the individual fluxes from about 40 of the brightest maser spots. Depending on the initial statistical realization of the turbulent velocity field that is created by the initial random numbers, these fluxes can gradually change by either increasing or decreasing. They can reach maxima or minima and then change in the opposite sense. We thus judge that the computer code is adequate to describe the evolution of turbulence for the current investigation.

\section{RESULTS OF COMPUTATIONS OF IMAGES FOR THE $25 \mathrm{GHz}$ METHANOL MASERS}

As the first step in comparing with the observations, we examined the general appearance of the brightest spots and estimated their sizes. Altogether, we performed somewhat more than 10 computations for the evolution of the turbulent velocity field - each starting from a different, statistically random realization for the velocity field.

We find that the sizes of the spots in our model match the sizes of the observed spots when the amplification factor for the brightest spot is about $8 \times 10^{6}$, which corresponds to an optical depth of about 16. JGW97 report that the brightness temperature for strongest spot in their observations was about $3.3 \times 10^{7} \mathrm{~K}$. The brightness temperature of the background in our model is then required to be about $4 \mathrm{~K}$ and is consistent with conclusions of Paper I, in which the characteristics of the maser region as a whole were considered.

In Paper I, we found that the number of brightest spots depends strongly on the particular statistical realization of
TABLE 1

Parameters of Representative MODELS

\begin{tabular}{ccc}
\hline \hline No. & $\sigma_{t} / v_{\text {th }}$ & $\tau_{0}$ \\
\hline $1 \ldots \ldots \ldots \ldots \ldots \ldots$ & 4.557 & 36.15 \\
$2 \ldots \ldots \ldots \ldots \ldots \ldots$ & 4.801 & 35.00 \\
$3 \ldots \ldots \ldots \ldots \ldots \ldots$ & 4.700 & 34.90 \\
\hline
\end{tabular}

the initial turbulent velocity field. We have confirmed this conclusion in the current investigation by examining a much greater number of the brightest spots. The basic parameters of three representative initial realizations are given in the Table 1. In these, the numbers of spots that exceed a specified level of brightness are quite different. The essential properties of the turbulent velocity field, such as the steepness of the spectrum, the dispersion in the turbulent velocities, and the optical depth parameter $\tau_{0}$, for these models all have approximately the same values that were found adequate for $25 \mathrm{GHz}$ methanol masers in Ori-KL in Paper I. We limit our discussion to the three evolutionary sequences for the turbulence that begin from the set of realizations listed in Table 1.

The distances between the spots in the maps based on our model are uneven. In the turbulent medium, the spots should tend to form clusters (see, e.g., Gwinn 1994a). Such clusters are indeed evident in our maps. Maps similar to those of the current study also are given in Paper I. An example is the cluster around the brightest spot in realization 2 in Figure 1. The field of view in this figure is about 2" and contains several maser spots. This can explain why the maser spots from the JGS92 sample (at $3^{\prime \prime}$ resolution) split into several features at the higher angular resolution $(0 . \prime 07)$ of the JGW97 maps. The result is consistent with the images that were obtained with a smaller computational grid in Paper I.

An examination of the images of individual maser spots (which ordinarily are weaker than clusters) shows that they are surrounded by extended regions where the spectral properties of the radiation are similar to the spectral properties of the spot. See the example in Figure 2. This is reminiscent of the halos found around the spots of water masers (Gwinn 1994b) and around the spots of the brightest class II methanol masers (Minier, Booth, \& Conway 2002). The extended regions that appear in our computations are considerably greater in size than these observed halos. A natural explanation for this difference is the much smaller amplification factors of the $25 \mathrm{GHz}$ masers. An explanation in terms of amplification factors is in agreement with the trend that the class II methanol maser halos are much greater than those surrounding the much brighter water maser spots. The extended regions in our computations merge in the clusters of spots. Because of this complication, we did not attempt a thorough study of their properties.

We also considered the dependence of the sizes of spots on their brightnesses. To do this, we measured the maximum extent of maser spots at the flux that is $20 \%$ of that of the brightest point of the spot. The results of our measurements are given in Figure 3. It is clear that the average sizes of the spots increase as the spots become weaker. This is in agreement with the JGW97 observational data. There is 


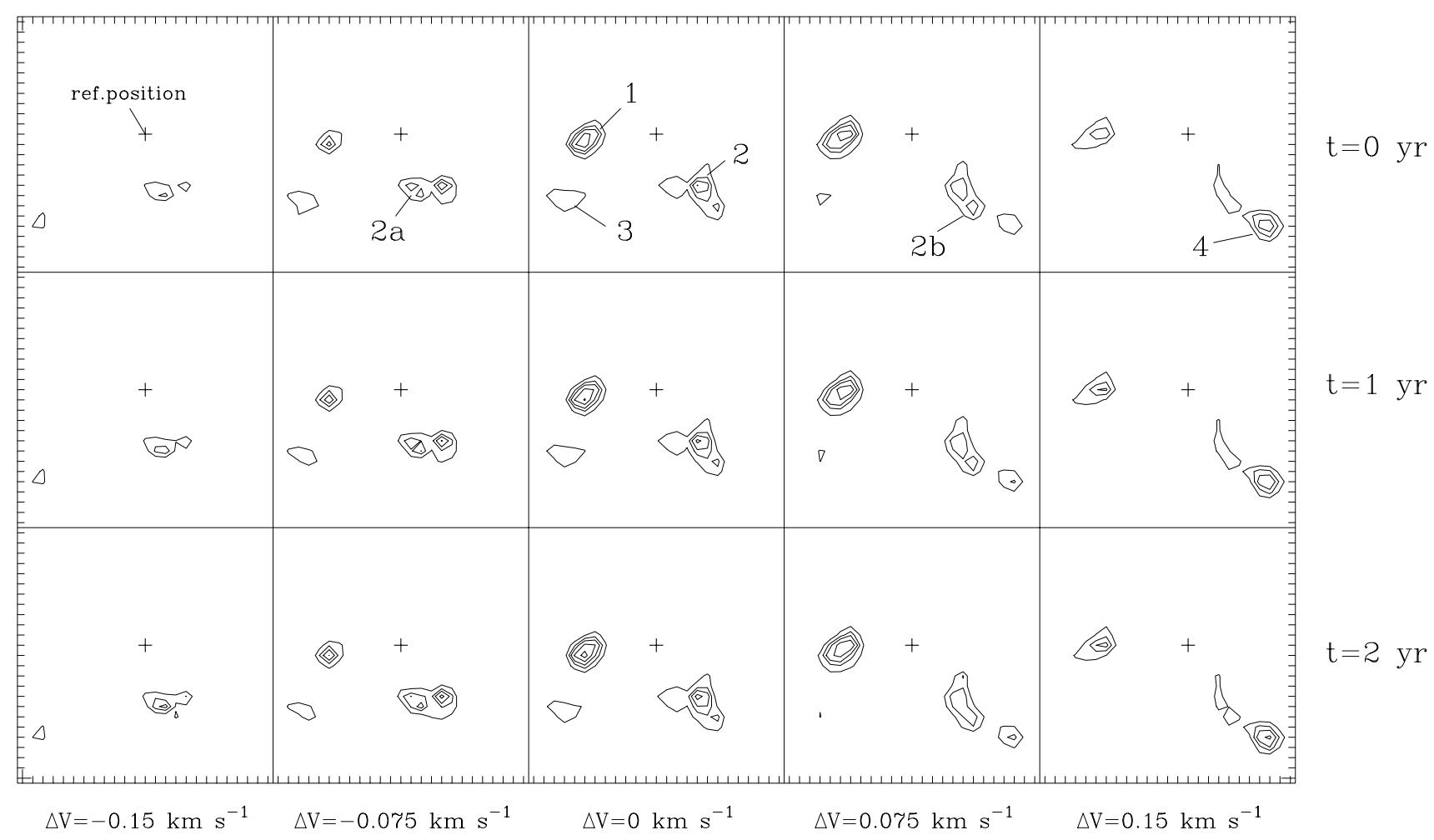

FIG. 1.-Images are shown in the vicinity of the brightest spot when the evolution of the turbulence in the slab starts from statistical realization 2. The size of an individual panel is about $2^{\prime \prime} \times 2^{\prime \prime}$. The panels in a particular column are at different frequencies as indicated by the shifts $\Delta V$ in Doppler velocity. The panels in a particular row show the evolution of the images with time. In all of the panels, contours are drawn at $0.4,0.5,0.6,0.7,0.75$, and 0.9 of the peak intensity. These images demonstrate how clusters of maser spots can be created in a field of view. In agreement with the observations, changes in the images with frequency and time are clearly seen. See $\S \S 4$ and 5 of the text for a discussion of the features marked with numbers.

0

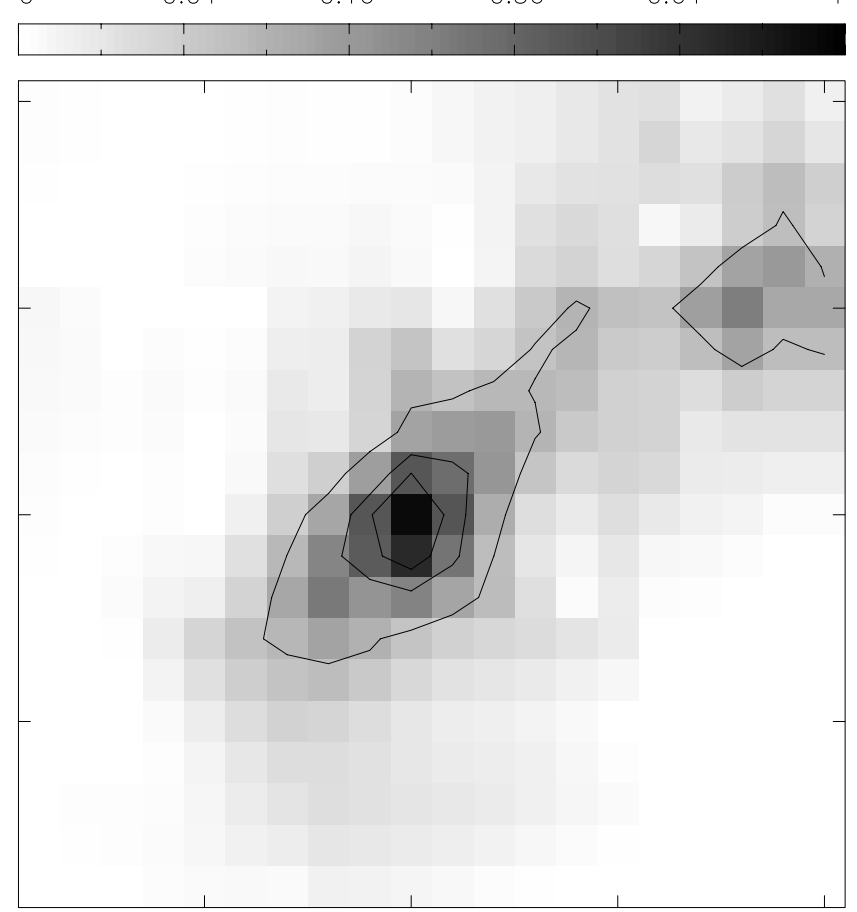

FIG. 2.-Example of the image of a spot and the surrounding extended region at the frequency for which the spot is brightest, for the random realization 1 . The calibration of the grey scale is given as a fraction of the peak intensity of the spot. The entire map corresponds to about 1 ". $6 \times 1$ "."6. Contour lines are drawn where the intensity is $0.1,0.3$, and 0.5 of the peak intensity. significant scatter due to the random character of the turbulent velocity field. The scatter is quite pronounced in the observational data as well. The slope of the relationship between the sizes of spots and their brightnesses carries information about the amplification factor of the masers. However, such estimates are subject to large uncertainty because the scatter is significant and the number of spots is limited.

In Figure 1, we show how the spot image depends on the Doppler velocity $V_{\mathrm{lsr}}$. Our calculations have better spectral resolution than the resolution of the JGW97 observations. We also use the ability of the computations to produce images for specific values of $V_{\mathrm{lsr}}$, whereas the observational data can only cover intervals of velocity. In agreement with the data of JGW97, there are significant changes in the images of the spots for values of $V_{\text {lsr }}$ that differ by $0.15 \mathrm{~km}$ $\mathrm{s}^{-1}$. These include the spatial shift of the peak emission for the spot 1 and the appearance of the new spot 4 that would be in an adjacent spectral channel in the observations. In order to provide information for comparisons with observational data that may be acquired in the future with better spectral resolution, we present images for intermediate values of $V_{\mathrm{lsr}}$. The difference in the spatial distribution of the maser emission can be seen to be considerable for a separation in $V_{\mathrm{lsr}}$ that is one-half of that of the velocity resolution for the current observational data. This difference occurs because of the presence of the spots with the narrow line breadths (e.g., spots $2 \mathrm{a}$ and $2 \mathrm{~b}$ ) and the existence of extended maser regions (e.g., 3) with or without a pronounced central spot. 


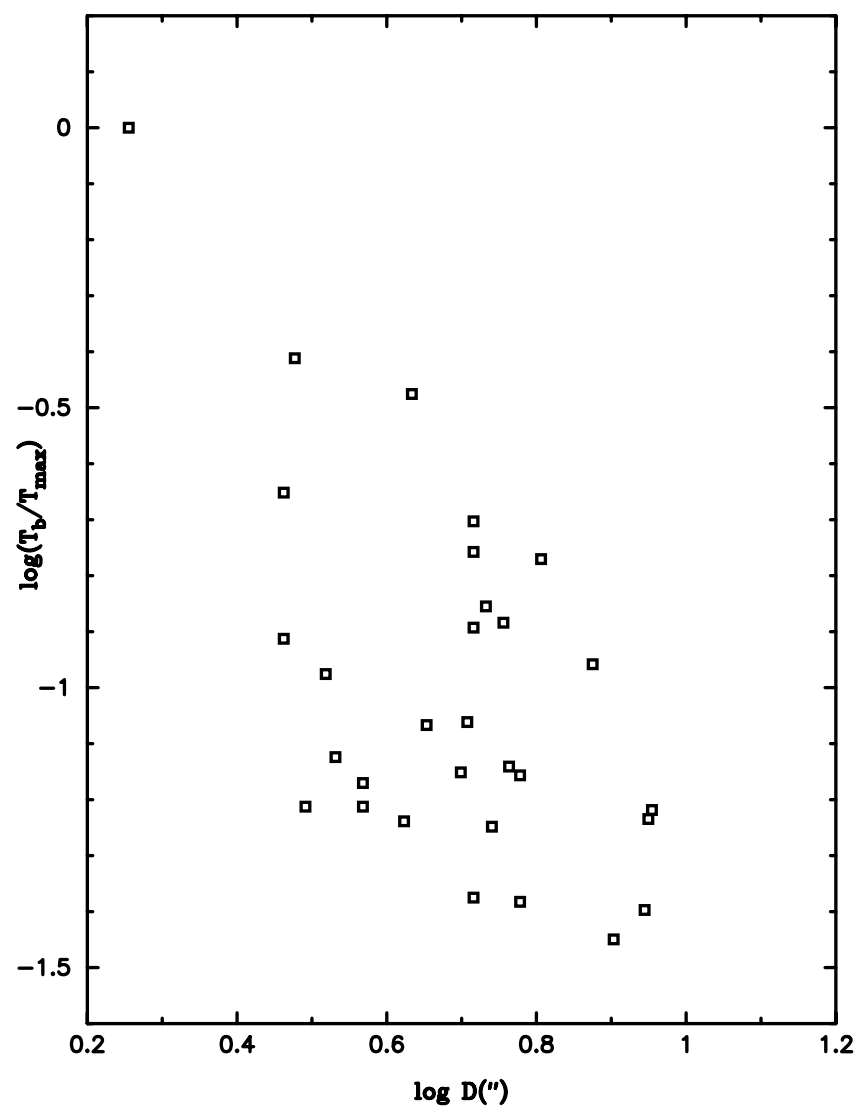

FIG. 3.-Relationship between the peak brightness temperatures and sizes of maser spots for the random realization 2 . Our $T_{\max }$ is the highest brightness temperature in the map. In general agreement with the observational data, there is significant scatter and the average sizes of the spots increase as the spots become weaker.

\section{RESULTS OF COMPUTATIONS FOR THE TIME VARIABILITY OF THE $25 \mathrm{GHz}$ METHANOL MASERS}

The main results of our studies for the time variability of the representative set of models are presented in Figures 1 and 4 . We find that the spots in our models display time variability over times of about $1 \mathrm{yr}$ - in agreement with observational data of JGW97. As in the observational data, the changes in the brightnesses of most of the maser spots are small. The main patterns of the time variability of the images of the spots are shown in Figure 1. For example, spots 1 and 2 are increasing in brightness while spot 3 undergoes a slight decrease in brightness. The pair of maser spots designated as 2a merge and the peak in the emission shifts to one of the spots. Examples of spots that split also are present in our computations. Indications of changes in the spectra of the spots are seen in Figure 1.

The brightest spot in a model can show noticeable variability (see the models involving realizations 1 and 3 , in which yearly changes of the brightest spot flux are $23 \%$ and $35 \%$, respectively), or it can remain at about the same brightness (realization 2) on the timescale of a year. We note that the variation in the brightness of the brightest spot can be significant for realizations in which the numbers of bright spots are quite different (e.g., see results for realizations 1 and 3 in Fig. 4). The weaker spots in Figure 4 also can display noticeable variability on a timescale of a year - in agreement with the JGW97 observations.
We also considered time variations of the integrated spectrum - that is, in the spectrum of emission from the region as a whole. The results are presented in the right-hand panels of Figure 4, where it can be seen that the integrated spectrum does not vary greatly on a timescale of a year. In the considered realizations the changes in the integrated spectrum were always less than $4 \%$ in a year. This is in agreement with the observational data of Menten et al. (1988) and is true for all of the realizations that we have considered, regardless of the number of bright spots. However, variations in the integrated spectrum do exist and the total flux can both increase (realization 1) or decrease (realization 2). This prediction of the computations can be checked by high sensitivity single dish observations separated by about 10 years.

The spatial resolution of our computations does not allow us to understand clearly how the integrated spectrum can remain nearly constant while the brightest spots vary considerably. However, our study does provide hints of an explanation for this phenomenon. First, we see extended regions with spectral properties similar to those of the associated spots (see Fig. 2). Second, the sizes of the spots increase with decreasing brightness (see Fig. 3). It is natural to suppose that the time variations in the spot brightnesses are accompanied by changes in the opposite sense in the sizes of the spots. The slope of the correlation between the brightnesses and the sizes of maser spots in our study is shallower than the one reported by JGW97. Most probably, this reflects the fact that we measure the sizes at a lower flux relative to the brightest point of the spot. Hence, more information on extended regions is included in our measurements. It thus seems likely that variations in the flux and spectral properties of the regions surrounding maser spots compensate to some extent for the variations in the brightnesses of the spots, and allow the integrated spectrum to be less sensitive to changes with time. Additional refined observations of spots and the extended regions surrounding them are necessary to elucidate this issue.

\section{CONCLUSIONS}

The computations here demonstrate that the basic properties of the images of the $25 \mathrm{GHz}$ methanol masers in OMC-1 can be reproduced by a model in which the masers are created in a turbulent slab of gas seen edge-on. More specifically, the computations show that (1) the features that appear to be single spots with the $3^{\prime \prime}$ beam are resolved into several spots in observations with 0 ".07 beam, (2) maser images show significant changes with frequency even for the cases when the frequencies are separated by less than a thermal breadth, (3) the average sizes of the spots increase as the spots become weaker while the relation between the peak intensity and the size of the spot shows substantial scatter, and (4) maser hot spots are surrounded by extended regions which emit at frequencies close to those of the associated hot spots.

Time-dependent computations show that the evolution of the turbulent velocity field can explain the observation that individual maser spots vary on a timescale of about a year, whereas the maser spectrum integrated over the entire masing region remains relatively constant. The computations predict that the changes in the integrated maser spectrum might become detectable in observations separated by about 10 years. 

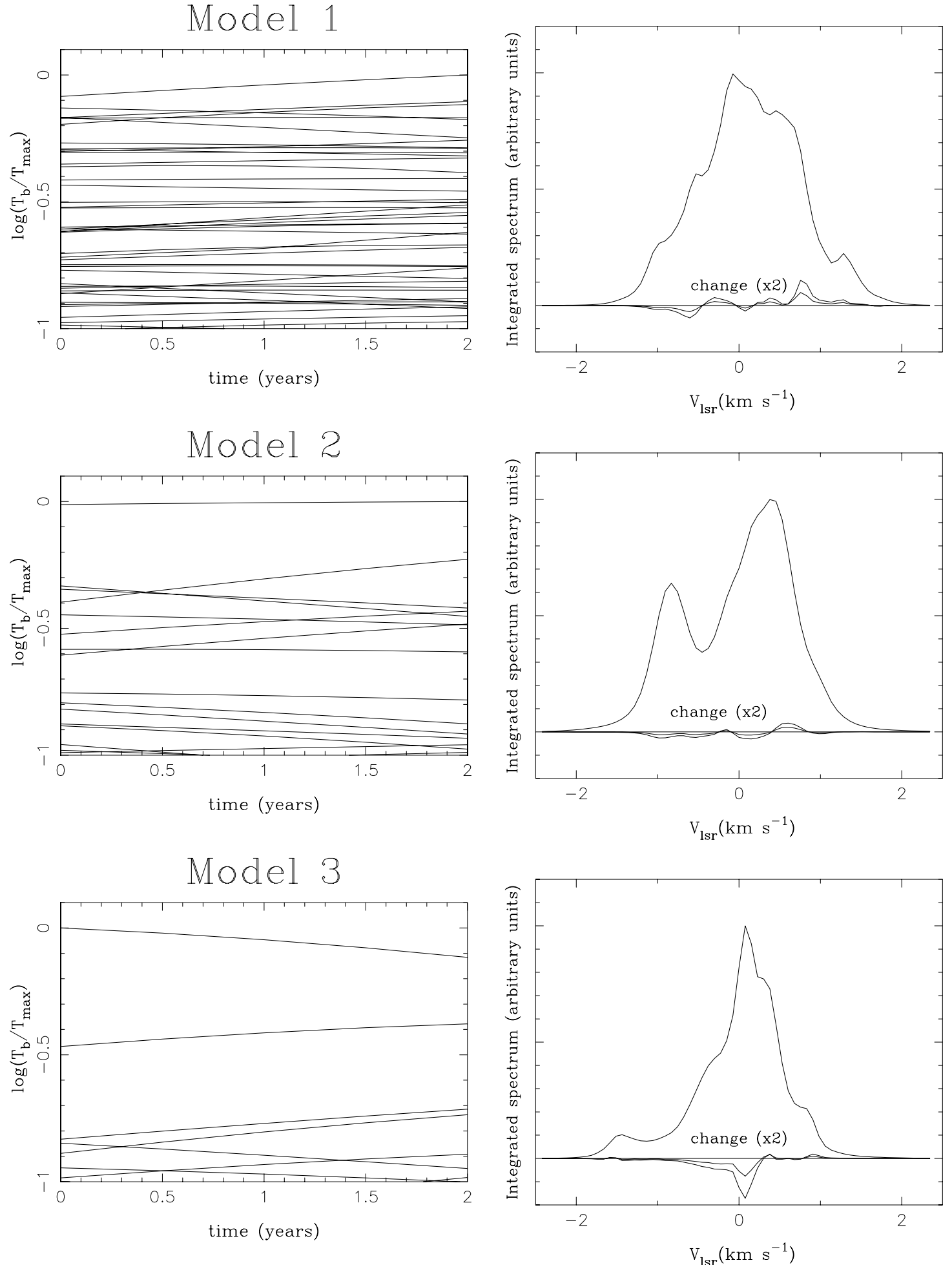

FIG. 4.-Modeling of the time variability. The left column of panels shows the variation of the peak intensities for individual maser spots for the evolution of the turbulent velocities starting from the initial configurations summarized in Table 1 . Note that these configurations have different numbers of bright maser spots. The right column of panels shows the initial spectra integrated over the entire maser region and the changes in these spectra after 1 and 2 yr. In agreement with the observations, the integrated spectra are nearly stable on the timescale of $1 \mathrm{yr}$, although some individual maser spots do change their brightnesses considerably within this period of time.

In this investigation, evidence is presented that the observational properties of the $25 \mathrm{GHz}$ methanol masers in OMC-1 can be understood through a consideration of the turbulent velocities alone. The basic parameters of the turbulent velocity field were taken to be the same as inferred in
Paper I. The modeling presented here and in Paper I shows that it may be possible to estimate the turbulent spectrum and the velocity dispersion in the regions of massive star formation on the basis of a consideration of the properties of masers. Observations of the $25 \mathrm{GHz}$ methanol masers in the 
other sources are highly desirable for further studies of the influence of turbulence on maser emission. Sensitive observations of the $25 \mathrm{GHz}$ masers, both single-dish and interferometric, are needed with high spectral resolution and with larger separations in time.

We do realize that the variations in the other physical quantities can be important for astrophysical masers. However, the goal of our investigation is to see how well turbulence alone can reproduce the observed characteristics of masers for a class of masers where there is evidence that the variation in the other physical quantities is small.

A. M. S. was supported by INTAS grant 97-11451 and federal program "Astronomy." W. D. W. acknowledges support from NSF grant AST 99-88104. We are grateful to H. W. Wyld for helpful discussions and providing the code for the evolution of turbulence based on Chen \& Shan (1992).
Chen, S., \& Shan, X. 1992, Comput. Phys. Commun., 6, 643

Deguchi, S. 1982, ApJ, 259, 634

Frisch, P. C. 1995, Space Sci. Rev., 72, 499

Gwinn, C. R. 1994a, ApJ, 429, 241 1994b, ApJ, 431, L123

Hills, R., Pankonin, V., \& Landecker, T. L. 1975, A\&A, 39, 149

Imai, H., Deguchi, S., \& Sasao, T. 2002, ApJ, 567, 971

Johnston, K. J., Gaume, R. A., Stolovy, S., Wilson, T. L., Walmsley, C. M., \& Menten, K. M. 1992, ApJ, 385, 232 (JGS92)

Johnston, K. J., Gaume, R. A., Wilson, T. L., Nguyen, H. A., \& Nedoluha, G. E. 1997, ApJ, 490, 758 (JGW97)

Kalenskii, S. V., Dzura, A. M., Booth, R. S., Winnberg, A., \& Alakoz, A. V. 1997, A\&A, 321, 311

Menten, K. M., Walmsley, C. M., Henkel, C., \& Wilson, T. L. 1986, A\&A, 157,318

\section{REFERENCES}

Menten, K. M., Walmsley, C. M., Henkel, C., \& Wilson, T. L. 1988, A\&A, 198,267

Minier, V., Booth, R. S., \& Conway, J. E. 2002, A\&A, 383, 614

Sobolev, A. M., \& Strel'nitskii, V. S. 1983, Soviet Astron. Lett., 9, 12

Sobolev, A. M., Wallin, B. K., \& Watson, W. D. 1998, ApJ, 498, 763 (Paper I)

Sutton, E. C., \& Herbst, E. 1988, ApJ, 333, 359

Turner, B. E. 1991, ApJS, 76, 617

Walker, R. C. 1984 , ApJ, 280, 618

Wallin, B. K., Watson, W. D., \& Wyld, H. W. 1998, ApJ, 495, 774 1999, ApJ, 517, 682

Watson, W. D., Wiebe, D. S., \& Crutcher, R. M. 2001, ApJ, 549, 377

Wiebe, D. S., \& Watson, W. D. 1998, ApJ, 503, L71

Zeng, Q., Lou, G. F., \& Li, S. Z. 1987, Ap\&SS, 132, 263 